
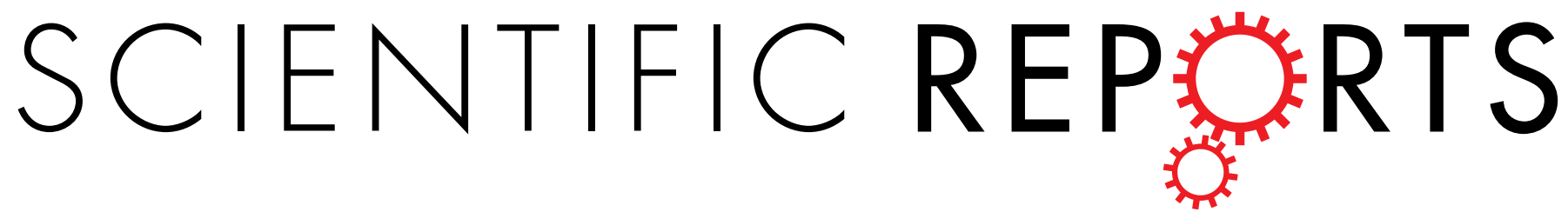

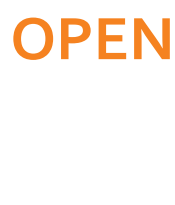

Received: 12 February 2016

Accepted: 27 May 2016

Published: 20 June 2016

\section{High Area Capacity Lithium-Sulfur Full-cell Battery with Prelitiathed Silicon Nanowire-Carbon Anodes for Long Cycling Stability}

Andreas Krause ${ }^{1,2}$, Susanne Dörfler ${ }^{3,4}$, Markus Piwko ${ }^{3}$, Florian M. Wisser ${ }^{4}$, Tony Jaumann ${ }^{5}$, Eike Ahrens ${ }^{5,6}$, Lars Giebeler ${ }^{5,6}$, Holger Althues ${ }^{3}$, Stefan Schädlich ${ }^{3}$, Julia Grothe ${ }^{4}$, Andrea Jeffery ${ }^{1}$, Matthias Grube ${ }^{1}$, Jan Brückner ${ }^{3}$, Jan Martin ${ }^{4}$, Jürgen Eckert ${ }^{5,6,7,8}$, Stefan Kaskel ${ }^{3,4}$, Thomas Mikolajick ${ }^{1,2,9}$ \& Walter M. Weber ${ }^{1,2}$

We show full Li/S cells with the use of balanced and high capacity electrodes to address high power electro-mobile applications. The anode is made of an assembly comprising of silicon nanowires as active material densely and conformally grown on a 3D carbon mesh as a light-weight current collector, offering extremely high areal capacity for reversible Li storage of up to $9 \mathrm{mAh} / \mathrm{cm}^{2}$. The dense growth is guaranteed by a versatile Au precursor developed for homogenous Au layer deposition on 3D substrates. In contrast to metallic $\mathrm{Li}$, the presented system exhibits superior characteristics as an anode in Li/S batteries such as safe operation, long cycle life and easy handling. These anodes are combined with high area density $\mathrm{S} / \mathrm{C}$ composite cathodes into a Li/S full-cell with an ether- and lithium triflatebased electrolyte for high ionic conductivity. The result is a highly cyclable full-cell with an areal capacity of $2.3 \mathrm{mAh} / \mathrm{cm}^{2}$, a cyclability surpassing 450 cycles and capacity retention of $80 \%$ after 150 cycles (capacity loss $<0.4 \%$ per cycle). A detailed physical and electrochemical investigation of the SiNW $\mathrm{Li} / \mathrm{S}$ full-cell including in-operando synchrotron $\mathrm{X}$-ray diffraction measurements reveals that the lower degradation is due to a lower self-reduction of polysulfides after continuous charging/discharging.

Lithium-sulfur batteries are pointed out as one of the most promising systems for advanced application in automotive or stationary electrical energy storage. The electrode components are predicted as lower in price and naturally highly abundant and therewith uncritical for shortage of resources. Introduction into the market has been prevented by limited stability and capacity as well as the absence of a suitable electrolyte when using full-cells with carbon-sulfur cathode, as carbonate-based electrolytes cannot be used due to their reaction with the polysulfides. Further on, present Li/S batteries employ a metallic Li foil as anode material resulting in undesired formation of cell safety critical Li dendrites. The use of a metallic $\mathrm{Li}$ anode can result in the loss of active material at the positive electrode, enhanced reactions with the electrolyte or short-circuiting of anode and cathode through the separator ${ }^{1}$. These points are addressed as key factors for high battery performance, long lifetime and safety. Silicon is one of the most promising anode materials replacing metallic Li due to a maximum specific capacity of $3579 \mathrm{mAh} / \mathrm{g}$ at room temperature ${ }^{2}$ but is accompanied by a large volume change upon lithiation and de-lithiation. To overcome stress related fracturing, several Si-based nanostructures ${ }^{3}$ that by their intrinsic geometry and neighboring free expansion volume allow an enhanced mechanical stress relaxation, like nanoparticles ${ }^{4-6}$, nanowires ${ }^{7-13}$,

${ }^{1}$ Namlab gGmbH, 01187 Dresden, Germany. ${ }^{2}$ Center for Advancing Electronics Dresden (CfAED), TU Dresden, Dresden, Germany. ${ }^{3}$ Fraunhofer Institute for Material and Beam Technology (IWS), Winterbergstr. 28, 01277 Dresden, Germany. ${ }^{4}$ Department for Inorganic Chemistry I, TU Dresden, Dresden, Germany. ${ }^{5}$ Leibniz Institute for Solid State and Materials Research (IFW) Dresden e.V., Helmholtzstr. 20, 01069 Dresden, Germany. ${ }^{6}$ Institut für Werkstoffwissenschaft, TU Dresden, Helmholtzstr. 7, 01069 Dresden, Germany. ${ }^{7}$ Erich Schmid Institute of Materials Science, Austrian Academy of Sciences, A-8700 Leoben, Austria. ${ }^{8}$ Department Materials Physics, Montanuniversität Leoben, Jahnstraße 12, A-8700 Leoben, Austria. ${ }^{9}$ Institute of Semiconductor and Microsystems Technology, TU Dresden, 01062 Dresden, Germany. Correspondence and requests for materials should be addressed to A.K. (email: Andreas.Krause@namlab.com) 

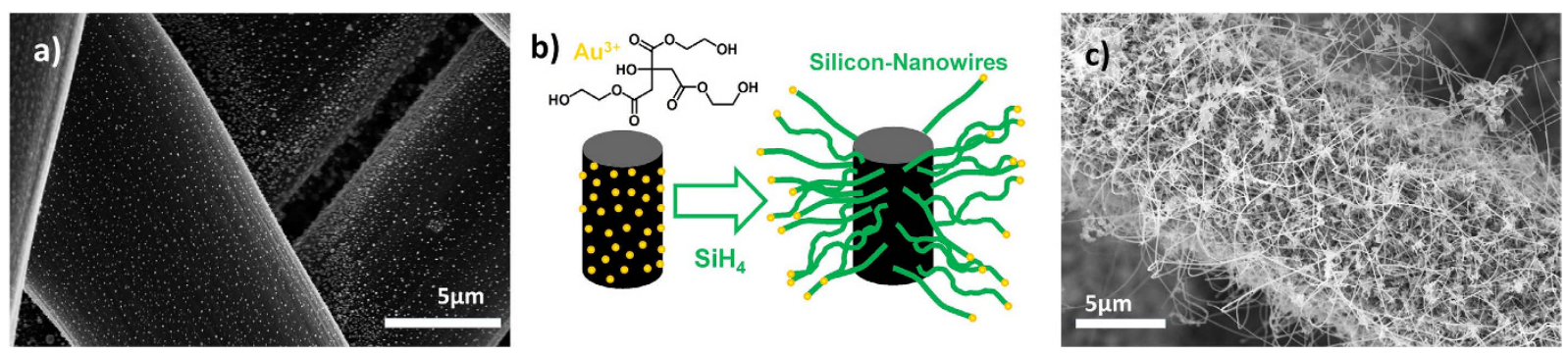

Figure 1. (a) Carbon fibers with three-dimensionally deposited Au nanoparticles. (b) Schematic view of the anode assembly based on the growth of Si nanowires on top of a single carbon fiber. The Au nanoparticles are the catalysts for the VLS growth of diluted $\mathrm{SiH}_{4}$ at $420^{\circ} \mathrm{C}$. (c) Carbon mesh completely covered with $\mathrm{Si}$ nanowires with a Si loading of more than $3.0 \mathrm{mg} / \mathrm{cm}^{2}$ prior to integration in Li half-cell.

microwires ${ }^{14}$ and nanotubes ${ }^{15-18}$, have been studied in battery setups. Further on, prelithiated Si nanostructures allow the insertion of $\mathrm{Li}$ independent of the chosen cathode material ${ }^{6,8,19,20}$.

As an ideal nanostructure to ensure both the possibility of free expansion and relaxation of Si as well as a good electrical contact to a carbon current collector, pure Si nanowires (SiNWs) have been investigated in half-cell setups as a promising solution to address both issues ${ }^{12,21}$. Recently, the SiNWs are grown by using planar sputtered Au layers to create Au nanoparticles (NPs) on a 3D surface ${ }^{11}$. A 3D SiNW growth with commercially available $\mathrm{Au}$ NPs has been reported for a $2^{\text {nd }}$ generation $\mathrm{Li}$ ion battery setup with $\mathrm{LiFePO}_{4}$ cathode and $\mathrm{LiPF}_{6}$ electrolyte with a maximum capacity of $100 \mathrm{mAh} / \mathrm{g}_{\mathrm{LiFePO}}{ }^{21}$. Nevertheless, Si appeals to be used as anode in high capacity lithium-sulfur systems since its high theoretical specific capacity is related to an insertion of 15 mole Li to 4 mole $\mathrm{Si}\left(\mathrm{Li}_{15} \mathrm{Si}_{4}\right)$. This high Li content compared to carbon with 1 mole $\mathrm{Li}$ per 6 mole $\mathrm{C}$ helps to build thin electrodes and to allow an easier balancing of the battery cell with excess Li of more than $2000 \%$ related to the sulfur mass ${ }^{22}$.

In this publication, we present a balanced Li/S battery with high capacity and long cycling stability using a prelithiated SiNW/C composite as anode material $\left(6.0 \mathrm{mAh} / \mathrm{cm}^{2}\right)$ and a S/C composite for the positive electrode $\left(2.4 \mathrm{mAh} / \mathrm{cm}^{2}\right)$ to overcome the battery failure due to typical Li dendrite formation resulting from state-of-the-art metallic Li foils. The Li excess in the prepared full-cell is limited to $200 \%$ of the cathode capacity, which allows an enhanced cycling stability ${ }^{22}$. Our full-cell shows with 460 cycles a 30 times higher cycling stability compared to previously reported $\mathrm{Li} / \mathrm{S}$ batteries with $\mathrm{SiNWs}^{7}$ and has a high specific capacity of $1.6 \mathrm{mAh} / \mathrm{cm}^{2}\left(714 \mathrm{mAh} / \mathrm{g}_{\text {sulfur }}\right)$ after 200 cycles. The conformal and heterogeneous integration of SiNWs with sufficient Si loading $\left(2.2 \mathrm{mg} / \mathrm{cm}^{2}\right)$ on the light-weight and conductive carbon mesh is the key element to achieve a high cycling stability and capacity. To enable the nanowire growth on the three-dimensional carbon network, a novel versatile gold 'Pechini' precursor was developed giving very homogenous and dense seed particle coverage. Physical analysis including in-operando synchrotron x-ray diffraction (SXRD) elucidates the mechanisms for the cell stability. The integration of SiNWs on carbon meshes is a highly promising approach to achieve high capacity batteries without elemental Li in combination with sulfur cathodes and ether-based liquid electrolytes to gain significantly improved cycling stability compared to known samples from literature ${ }^{7,23}$.

For the previously addressed tasks, the manuscript is divided into three parts. In the first part, morphology and crystallinity of the SiNW/C composite was analyzed before integration in a battery setup. The second part discusses the electrochemical investigations of the $\mathrm{SiNW} / \mathrm{C}$ composite in half-cell assemblies vs. $\mathrm{Li} / \mathrm{Li}^{+}$with subsequent in-operando as well as post-mortem characterization of morphology and crystallinity to elaborate the basic capacity fading mechanisms. The third part covers the integration of SiNW/C anodes in high capacity balanced Li/S full-cells by means of in-operando synchrotron X-Ray diffraction (SXRD) measurements and detailed electrochemical characterization.

\section{Results and Discussion}

Structural characterization of the as-grown SiNWs. The preparation of the SiNW/C composite electrodes is depicted in Fig. 1. The SiNWs are grown selectively with the prepared Au nanoparticles as catalyst seed (Fig. 1a) via the vapor-liquid-solid (VLS) mechanism and $\mathrm{SiH}_{4}$ as the $\mathrm{Si}$ precursor gas on the carbon mesh. A detailed description of the novel Au nanoparticle fabrication can be found in the Suppl. Info. (Figs S1-3). Figure $1 \mathrm{~b}$ schematically describes the VLS growth of SiNWs on the carbon fibers. Figure 1c presents a representative sample with very high SiNW loading of more than $3 \mathrm{mg} / \mathrm{cm}^{2}$ on a single carbon fiber. The amount of $\mathrm{Si}$ is reproducibly tuned by deposition time as well as $\mathrm{SiH}_{4}$ dilution in $\mathrm{H}_{2}$ to optimize the $\mathrm{Si}$ content of the negative electrode composite shown in Krause et al. ${ }^{24}$. Comparable SiNW loadings on carbon meshes have been recently reported by Peled et al. ${ }^{21}$ using commercially available Au nanoparticles. Nevertheless the development of a stable and recyclable $\mathrm{Au}$ precursor enables a resource efficient gold catalyst seed application on scalable substrates in this work. For the assembly of a well-balanced full-cell, a SiNW/C composite as negative electrode can be prepared matching the high S/C positive electrode composite.

The convenient sample structure enables an excellent insight with $\mathrm{x}$-ray diffraction analysis. Figure 2 summarizes the structural properties of each step of the SiNW/carbon composite electrode preparation prior to lithiation with X-ray powder diffraction measurements. The bare substrate shows the signature of an amorphous carbon layer with a broad signal with a maximum at $2 \theta=51^{\circ}$ (Fig. 2a). After the deposition of the Au layer on the carbon fibers and the formation of Au nanoparticles on the surface, reflections appear in the pattern (Fig. 2b) 


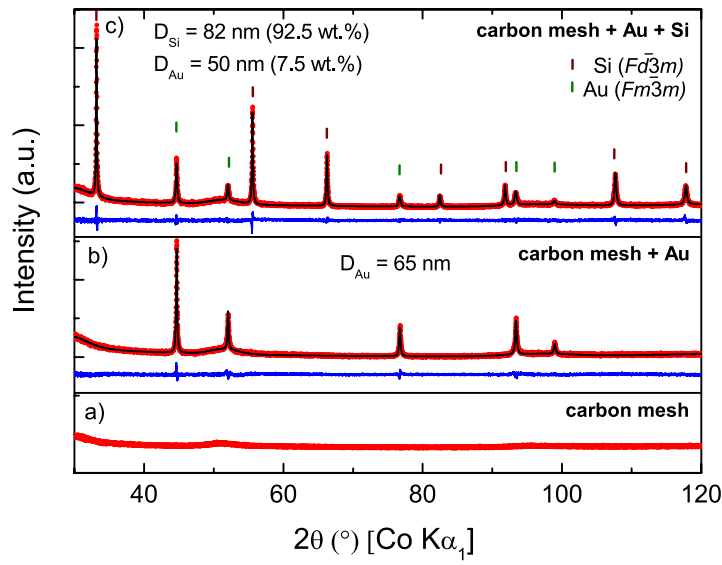

Figure 2. XRD pattern (red) of the carbon mesh (a), the Au-treated carbon mesh (b) and the final SiNWs (c). Crystallite sizes and phase contents of the crystalline phases are listed in the graphic determined by a Rietveld analysis. The result of the Rietveld analysis is depicted as the calculated pattern in black. The difference between calculated and measured pattern is plotted in blue.

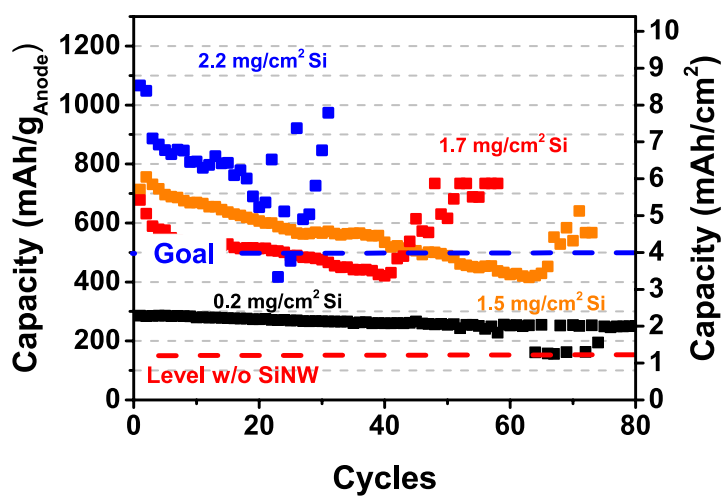

Figure 3. Cycling Stability of anodes of SiNW in carbon mesh versus lithium for different Si loadings. The $1^{\text {st }}$ cycle of a cell with $2.2 \mathrm{mg} / \mathrm{cm}^{2}$ SiNW loading shows an excellent capacity of up to $9 \mathrm{mAh} / \mathrm{cm}^{2}$.

corresponding to the cubic structure of Au with space group $F m \overline{3} m$. After the growth of the SiNWs, distinct reflections of crystalline $S i$ with the space group $F d \overline{3} m$ appear while Au becomes the minor phase as expected (Fig. 2c). An averaged Au crystallite size of $65 \mathrm{~nm}$ is determined for $\mathrm{Au}$, which is decreased to approximately $50 \mathrm{~nm}$ after SiNW growth. The observed crystallite size for the crystalline $\mathrm{Si}$ is calculated to $82 \mathrm{~nm}$ in average. High-resolution TEM bright field images of the samples with as-grown crystalline SiNWs are shown in the Suppl. Info. (Fig. S4) including EDXS analysis (Fig. S5).

Electrochemical characterization of SiNW electrodes versus lithium. Prior to integration in Li/S full-cells, half-cells with SiNW/C composite electrodes vs. elemental Li are studied in the ether- and lithium triflate-based electrolyte to understand the electrochemical behavior of SiNW electrodes. The electrochemical test of the composite as positive electrode reveals outstanding high areal capacities up to $9 \mathrm{mAh} / \mathrm{cm}^{2}$ (Fig. 3). When compared to the areal capacity of a pure carbon mesh $\left(1.2 \mathrm{mAh} / \mathrm{cm}^{2}\right)$, SiNWs drastically increase the areal capacity due to the high theoretical gravimetric capacity of $\mathrm{Si}\left(3579 \mathrm{mAh} / \mathrm{g}_{\mathrm{Si}}\right.$ for $\left.\mathrm{Li}_{15} \mathrm{Si}_{4}\right)$. For practical applications of $\mathrm{Li} / \mathrm{S}$ cells, an areal capacity of $6.0 \mathrm{mAh} / \mathrm{cm}^{2}$ is required to reach energy densities of commercial lithium-ion batteries ${ }^{25}$. This outstanding high value is reproducibly exceeded by the SiNW composite anodes presented in this work. Note that the presence of Au particles makes a negligible contribution to the charge capacity, e.g. shown by Chan et al. ${ }^{26}$. To further reduce costs, SiNWs can also be grown with many different catalyst metals, such as $\mathrm{Cu}$, $\mathrm{Ti}, \mathrm{Al}$, In etc. shown by Schmidt et al. ${ }^{27}$.

Figure 3 also depicts that an increase in SiNW mass loadings leads to lower cycle stability of the Li half-cells. The high capacities of the cells result in high charge/discharge currents when using a constant rate of $\mathrm{C} / 5$. It is known, that high currents promote Li dendrite formation of metallic Li and induce active material loss during cycling ${ }^{28-30}$. Other reasons of decreasing capacity or cell failure may be caused by electrolyte depletion due to an unstable $\mathrm{SEI}^{31-33}$ or due to the loss of electrical contact between the SiNWs and the carbon current collector during cycling. However, in the lithium-sulfur full-cells described later, several mechanisms can be disregarded due to the absence of elemental Li. 

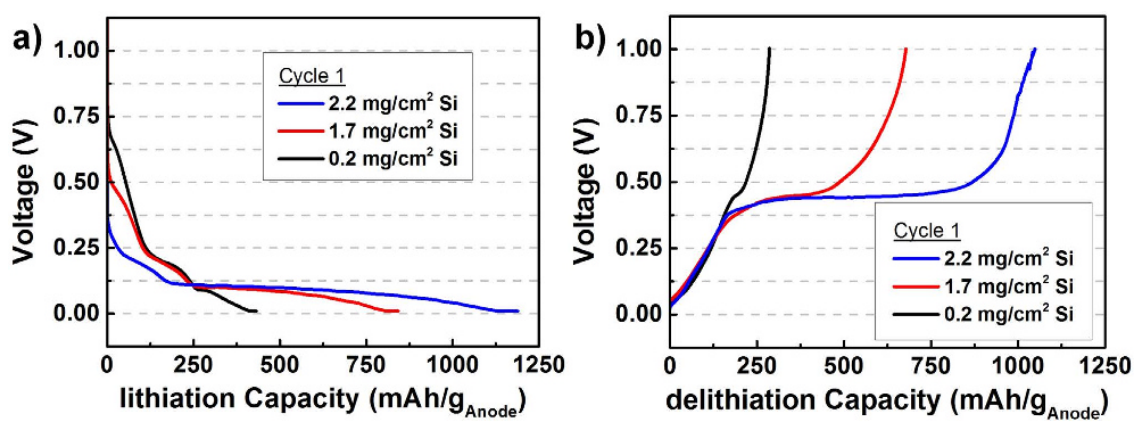

Figure 4. Voltage Profile in the 1st cycle using a carbon mesh with different mass loading of SiNWs in the halfcell setup during lithiation (a) and delithiation (b) of Si.

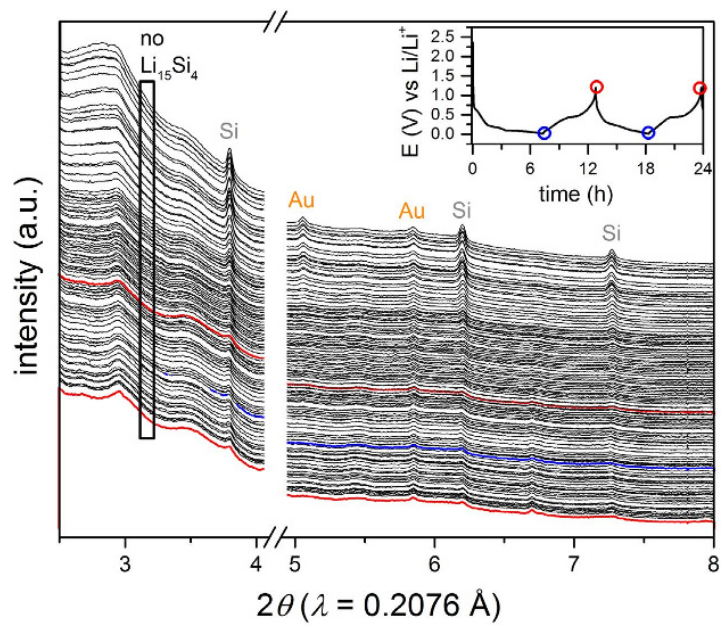

Figure 5. In-operando synchrotron powder diffraction data of the SiNW/C composite electrode cycled vs $\mathrm{Li} / \mathrm{Li}^{+}$(half-cell) at $0.5 \mathrm{~mA} / \mathrm{cm}^{2}$. Corresponding electrochemistry with assigned position of the colored XRD pattern (inset top right). Si and Au pattern diminish during first charging due to amorphization with $\mathrm{Li}$ insertion. Other peaks can be attributed to e.g. LiOH formation or inactive battery components.

The influence of different Si mass loadings on the capacity is derived from the voltage profiles of the half-cells in Fig. 4. Almost only the carbon influences the reachable capacity of up to $250 \mathrm{mAh} / \mathrm{g}$, when a carbon mesh with a low Si loading of $0.2 \mathrm{mg} / \mathrm{cm}^{2}$ is used. Higher Si loadings show a long flat voltage plateau at approximately $0.11 \mathrm{~V}$ during lithiation (Fig. 4a). The appearance of this plateau represents the formation of lithiated amorphous $\mathrm{Si}$, which is the lithiation potential of pure crystalline Si from SiNWs s4,35. The following gradual dropping to $0.01 \mathrm{~V}$ may be related to the lithiation to $\mathrm{Li}_{15} \mathrm{Si}_{4}$ as the lithium-richest compound to be electrochemically formed at room temperature ${ }^{2,36}$. The delithiation of the $\mathrm{Li}-\mathrm{Si}$ alloy is visible due to a plateau in Fig. $4 \mathrm{~b}$ at about $0.45 \mathrm{~V}$. The appearance of this plateau stands in particular for delithiation of $\mathrm{Li}_{15} \mathrm{Si}_{4}$ and the coexistence with an amorphous $\mathrm{Li}_{\mathrm{z}} \mathrm{Si}$ phase $\mathrm{p}^{2,35,36}$.

Structural characterization of cycled SiNWs electrodes. To study the degradation phenomena of the SiNW/C composite electrode, in-operando as well as post-operation analysis was carried out in the half-cell setup. In-operando SXRD was used to record the first cycles and observe the structural changes of the SiNWs as shown in Fig. 5. The Si reflections successively diminish distinctly depending on time related to an amorphization process at simultaneously increasing the Li content during charging. Some residues of crystalline Si and $\mathrm{Au}$ remain at the end of discharge as a result of incomplete lithiation owing to the relatively high current rate of $0.5 \mathrm{~mA} / \mathrm{cm}^{2}$ or insufficient electrical contact. These high current rates have been first reported for in-operando Si lithiation here, but are essential to understand degradation mechanisms for product-oriented application. No crystalline $\mathrm{Li}_{15} \mathrm{Si}_{4}$ is observed at the end of the lithiation process ${ }^{37}$, typically causing a degradation of the $\mathrm{Si}$ anodes $^{36,38,39}$. This result may explain the long-term cyclability of our samples ${ }^{40}$. As the SiNW showed continuous capacity loss at this current rate (Fig. 3), crystalline $\mathrm{Li}_{15} \mathrm{Si}_{4}$ is excluded as the major reason for degradation in our experiments.

After ten charge and discharge cycles, a coin cell was dismantled and the SiNW/C electrode (Si loading approx. $1.5 \mathrm{mg}_{\mathrm{Si}} / \mathrm{cm}^{2}$ ) was removed for analysis. The sample was rinsed in diluted $\mathrm{HNO}_{3}$ to remove electrolyte residues. Figure 6 shows the SEM images of this sample: Most of the nanowires are still attached to the carbon fibers. The images illustrate an intact electrical contact of the SiNWs with the substrate resulting in long cycling duration. 

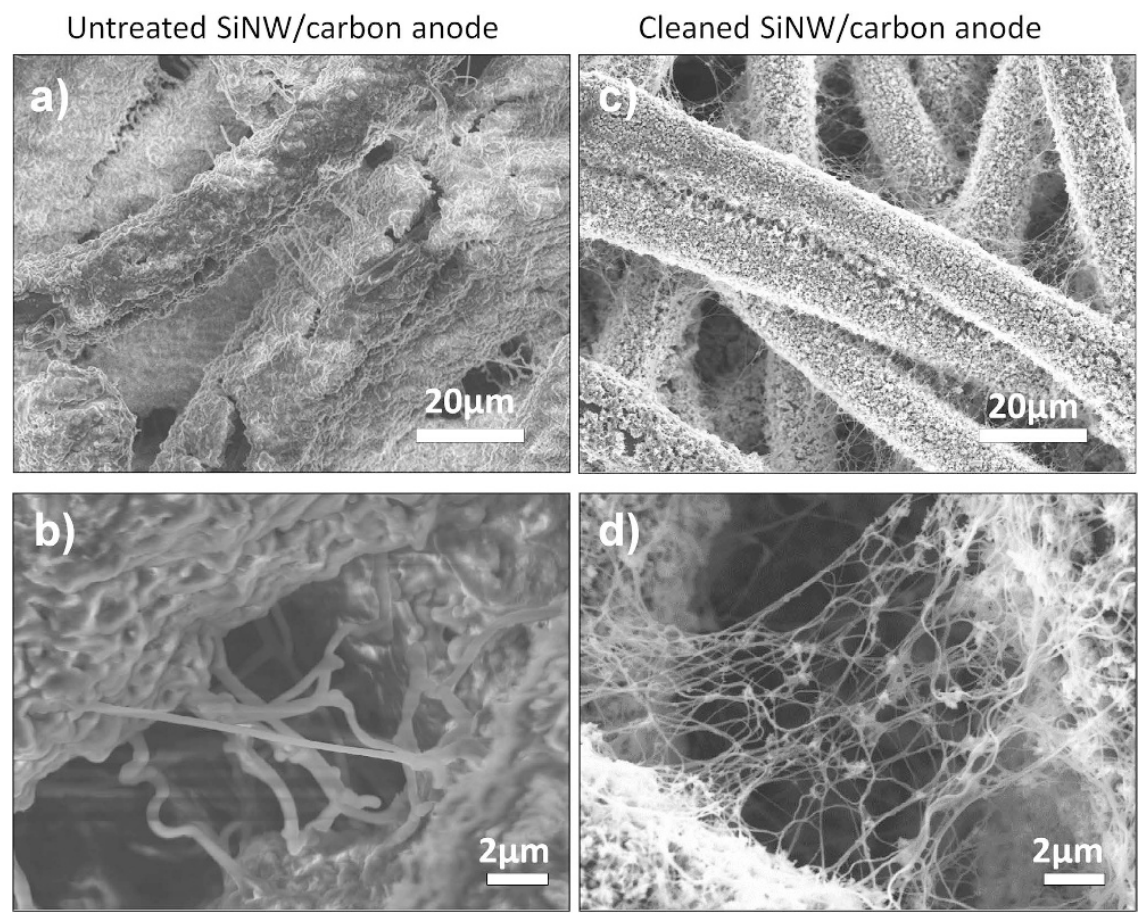

Figure 6. SEM investigation of cycled SiNW anodes. (a,b) A thick insulating SEI is formed on top of the carbon mesh and completely covers the SiNWs. (c,d) After removal of the SEI with diluted $\mathrm{HNO}_{3}$, free-standing nanowires are visible and are still attached to the carbon surface.

a)

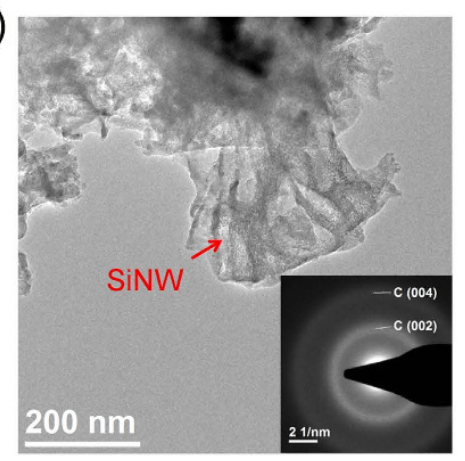

b)

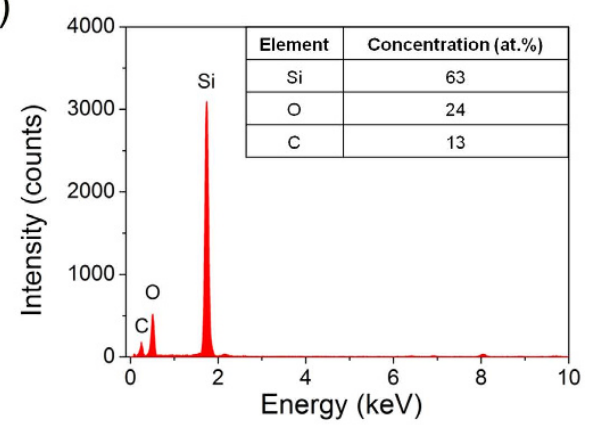

Figure 7. TEM analysis of the cycled SiNWs on the carbon support. (a) Bright-field images and SAED pattern and (b) the corresponding EDX measurement of the analyzed area.

This finding is in contrast to results presented by Chan et al. ${ }^{26}$ dealing with the hypothesis of cell failures due to structural disintegration of the SiNWs. The cycled sample shows undulated Si nanowires, which are attributed to the final relaxed amorphous state originating from the initially single crystalline Si nanowires. This bending upon lithiation starting with the first cycle is in accordance with literature $e^{41,42}$

Additional structural information of the cycled SiNW/C composite is shown in Fig. 7. Figure 7a depicts a bright-field TEM image of agglomerated SiNWs verified by the high concentration of Si in the EDX spectrum (Fig. 7b). The SAED patterns of the SiNW remains reveal no crystalline Si indicating amorphous Si. The complete amorphization of the crystalline SiNWs in the first lithiation cycles supports the in-operando measurements in Fig. 5. The SiNWs are considerably deformed as a result of the enormous volume change during cycling. High concentrations of oxygen are attributed to the exposure to air and the treatment with $\mathrm{HNO}_{3}$ to remove the interfering SEI after disassembling. The Debye-Scherrer rings in the SAED pattern in Fig. 7a represent both amorphous $\mathrm{SiO}_{2}$ as result of the etching process and amorphous carbon as it is characteristic for the supporting mesh.

Characterization of prelithiated SiNWs in a full-cell setup. Previous half-cell tests versus $\mathrm{Li} / \mathrm{Li}^{+}$help to identify the basic working principles of the anode material and allows to compare the results to literature. Recently, SiNW/C composite negative electrodes with comparable capacity have been reported for $\mathrm{LiFePO}_{4} 2^{\text {nd }}$ Generation batteries $^{21}$. In this work, prelithiated SiNW/C composite electrodes have been integrated in high 


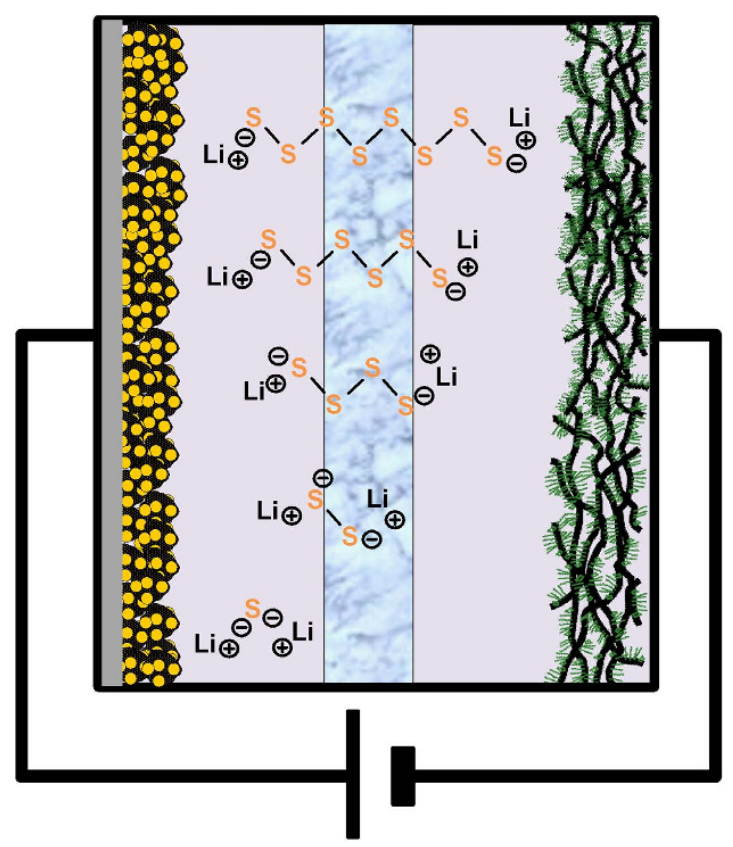

Figure 8. Scheme of $\mathrm{Li} / \mathrm{S}$ full-cell with $\mathrm{SiNW} / \mathrm{C}$ anode, $\mathrm{S} / \mathrm{C}$ cathode and separator.

capacity Li/S batteries to replace Li metal as only negative electrode with sufficient capacity. The antecedent prelithiation of SiNWs in a separate half-cell has the advantage to compensate the Li loss due to SEI formation in the first cycles ${ }^{19,20}$. Electrochemical testing of the composite electrodes in a Li/S full-cell exposes the real properties of the negative electrode material and provides the necessary data for introduction into application without the obstacle of Li dendrite formation. These results give valuable information primarily on the Li transport mechanism in Li/S cells.

In contrast to $\mathrm{Li}$-ion cells or to the $\mathrm{Si}$ - $\mathrm{Li}$ system, where only $\mathrm{Li}$ ions migrate between the electrodes, in $\mathrm{Li} / \mathrm{S}$ cells the charge is carried by lithium-polysulfides. During discharging, sulfur is dissolved by the formation of long chained polysulfides. After reduction of the chain length to at least $\mathrm{Li}_{2} \mathrm{~S}_{4}$, the final product of electrochemically-driven reaction is $\mathrm{Li}_{2} \mathrm{~S}$. During charging, lithium remigrates towards the negative electrode and sulfur is formed in the reverse reaction $^{43-45}$. A scheme of the used Li/S full-cell assembly with S/C cathode and SiNW/C anode is presented in Fig. 8.

In-operando synchrotron powder diffraction of prelithiated SiNW/S full-cell. In order to study the initial degradation phenomena, the SiNW Li/S full-cell was cycled for four times at a rate of 1/5 C and simultaneously diffraction patterns have been recorded every $10 \mathrm{~min}$. Conventional $\mathrm{Li} / \mathrm{S}$ half-cell batteries were investigated by in-operando diffraction previously ${ }^{46-49}$. Since the degradation of the Li metal electrode by migrating polysulfide species can considerably affect the degradation of $\mathrm{Li} / \mathrm{S}$ batteries ${ }^{22}$, an investigation of a sulfur positive electrode vs. lithiated SiNWs by in-operando XRD measurements is of vast interest. The electrochemical results and the corresponding synchrotron diffraction pattern are demonstrated in Fig. 9. The entire waterfall plot of the measurements is found in the supplementary information (Fig. S6).

Initially, $\alpha$-sulfur with space group $F d d d$ is present as typically stable phase at ambient temperature in the positive electrode composite (Fig. 9a green pattern). No distinct crystalline Si reflection is observed in the initial pattern after the pre-lithiation process (Fig. 5). During the first discharging, the $\alpha$-sulfur phase disappears as a result of the lithiation of sulfur and complete dissolution to polysulfides. At the end of the first discharge cycle $\mathrm{Li}_{2} \mathrm{~S}$ appears with a broad $\{111\}$ reflection. During recharging, the disappearing $\mathrm{Li}_{2} \mathrm{~S}\{111\}$ reflection indicates a reversed reaction from $\mathrm{Li}_{2} \mathrm{~S}$ to soluble polysulfides. Interestingly, at the end of the second charging, sulfur reconstitutes in its monoclinic $\beta$-allotrope with space group $P 2_{1} / c$. The structure model of $\beta$-sulfur was refined by the Rietveld method according to the diffraction data (see Supp. Info. in Fig. S7). At this stage, the entire process is highly reversible over at least four cycles with the final products $\mathrm{Li}_{2} \mathrm{~S}$ and $\beta$-sulfur. No other crystalline phases are present in the investigated diffraction range. The results agreed with previously reported results for Li/S batteries ${ }^{46-48}$. The reflection intensities of $\mathrm{Li}_{2} \mathrm{~S}$ and $\beta$-sulfur remain almost unchanged in each cycle which points to low self-discharge and low sulfur consumption on the Si negative electrode surface. This finding may be attributed to the lower reactivity of Si and its SEI compared to Li metal as shown for Si nanoparticles by Jaumann et al. ${ }^{4}$. Importantly, this data supports the fact that the lower self-reduction of polysulfides results in a lower degradation of our full-cell setup highlighting the relevance of this full-cell for real application.

Cycling stability of SiNW/C anode vs S/C cathode. To examine the long-term properties of lithiated SiNWs, a separate full-cell-test versus a sulfur positive electrode $\left(2.4 \mathrm{mAh} / \mathrm{cm}^{2}\right)$ was carried out. Chakrapani et al. showed that limiting the anode capacity promotes stable cycling performance 9 . Therefore, carbon meshes with a moderate $\mathrm{Si}$ loading of approximately $1.5 \mathrm{mg}_{\mathrm{Si}} / \mathrm{cm}^{2}\left(6.0 \mathrm{mAh} / \mathrm{cm}^{2}\right)$ were chosen as anode materials for a balanced full-cell. Figure 10 compares the cycling performance of the sulfur positive electrode versus 


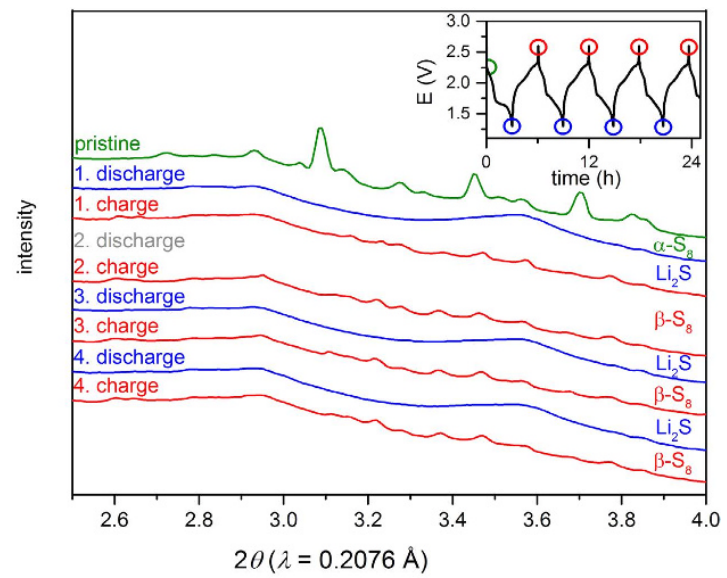

Figure 9. In-operando synchrotron powder diffraction data of the prelithiated SiNWs cycled vs S (full-cell) at $1 / 5 \mathrm{C}$. Corresponding electrochemistry with assigned position of the colored XRD pattern (inset top right). The diffraction pattern distinctly changes during the $1^{\text {st }}$ charge/discharge cycle from $\alpha$-sulfur to $\beta$-sulfur.
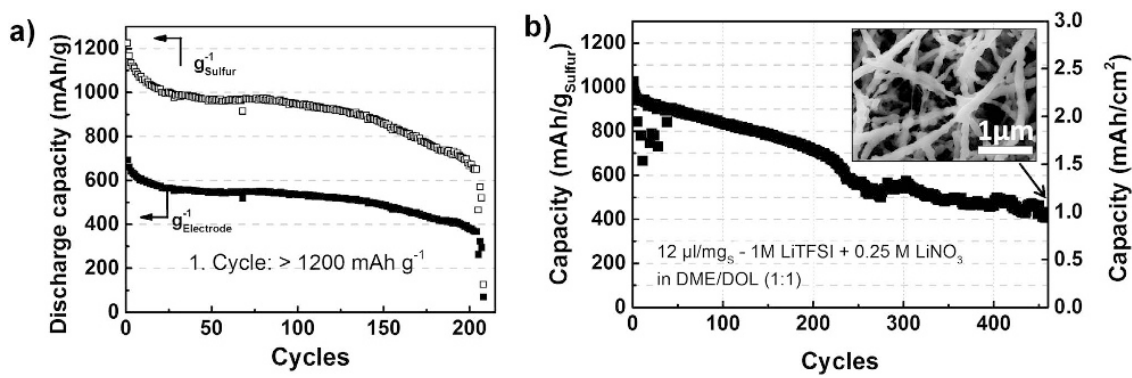

Figure 10. Comparison of the cycle performance of sulfur cathode versus (a) Li foil with cell failure after 200 cycles due to Li dendrite formation and (b) including SiNW anode, which show slow capacity decay but without complete cell failure up to 460 cycles. (Inset) SEM image of SiNW/C anode after 460 cycles.

(a) Li and (b) the SiNW/C composite anode. Note that the capacity of the SiNW Li/S full-cell is limited by the $\mathrm{S} / \mathrm{C}$ composite cathode due to the excess of Li from the prelithiated SiNWs. The capacity of $1027 \mathrm{mAh} / \mathrm{g}_{\text {sulfur }}$ corresponds to an energy density of $410 \mathrm{Wh} / \mathrm{kg}$ including the active components, the current collector and the separator. The excess of Si plays only a minor part in the energy density of the full-cell. Because of the high specific capacity of $\mathrm{Si}$, a small extra amount of $\mathrm{Si}$ results in a remarkably higher negative electrode capacity, while the change to the weight is negligible compared to the other components.

Until cycle 150 the metallic lithium and the SiNW anodes show a similar capacity, with two exceptions. The first difference is faster degradation of cells with Li negative electrode during the first 25 cycles, possibly due to formation of Li dendrites or irreversible polysulfide dissolution. The second difference is the appearance of a capacity plateau with a metallic lithium anode until cycle 150, while SiNW anodes exhibit a gradual degradation with increasing number of cycles. This behavior is most likely caused by an irreversible electrolyte decomposition and continuous SEI formation at the SiNW surface. A progressive degradation of electrical contact of the SiNWs during lithium insertion has not been observed in the first cycles in Figs 5 and 6. As it is shown in Fig. 10b (Inset), the stability of the nanowire structures on carbon is assured beyond 460 cycles in the Li/S full-cell. Nevertheless, loss of electric contact as fading mechanism cannot be explicitly excluded in the long term measurements.

After 150 cycles the degradation of the cells with elemental Li foil as anode is accelerated until complete breakdown and cell failure occurs due to Li dendrite formation causing a short circuit between the electrodes. In the case of the full-cell with a SiNW-based anode, the short circuit is avoided even with fading capacity. After complete loss of SiNW capacity, the carbon mesh still can reversibly insert Li ions. Since the carbon mesh contributes with approx. $1.2 \mathrm{mAh} / \mathrm{cm}^{2}$ to the overall capacity (Fig. 3), the cell capacity is mainly shaped by the electrochemical property of carbon. For a description of the long cycling performance of carbon vs. a sulfur positive electrode, the reader is forwarded to Brückner et al. ${ }^{22}$.

The voltage profiles in Fig. 11 demonstrate the contribution of the carbon mesh during lithiation and delithiation. While the first discharge curve is characterized by a flat plateau at approximately $1.58 \mathrm{~V}$, corresponding to the delithiation of $\mathrm{Si}$, the plateau extension is reduced with every further cycle and the cell

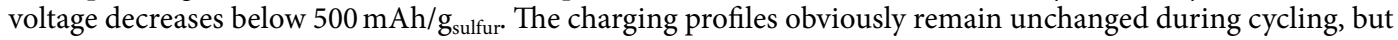
an increased charge voltage may result from a loss of active material or the presence of an insulating layer on the electrode surfaces. Discharging as well as charging profiles at cycle 300 indicate that the carbon mesh is still 

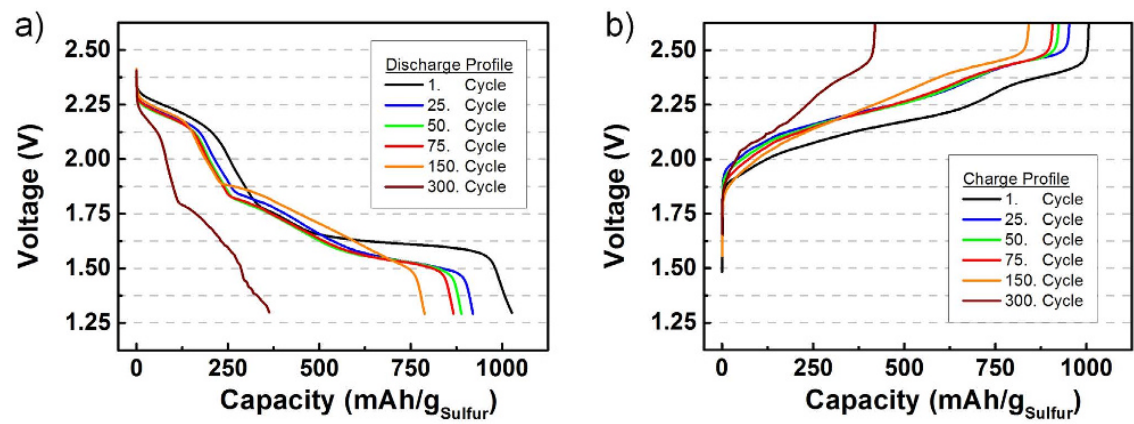

Figure 11. Voltage profiles for different cycles of lithium-sulfur-cell with SiNW anode: (a) discharge profiles and (b) charge profile.

working as an intercalation negative electrode for lithium ions and thereby allows a continuously and stable operating lithium-sulfur battery.

Even if the carbon mesh plays an important role in a Li/S full-cell using SiNW in carbon as an anode material, the advantages of $\mathrm{Si}$ are obvious:

- The anode capacity is significantly enhanced above pure graphite capacity for over 450 cycles.

- The use of potentially dangerous lithium is avoided.

- Cell failure due to lithium dendrite formation short-circuiting the electrodes is avoided.

- There is no need for a copper current collector, which reduces the inactive material mass of the cell.

The Li/S battery is limited by the S/C cathode not by the prelithiated SiNW/C composite anode as the anode provides an excess of Li for full charge/discharge cycles. The full-cell uses a standard setup with a commercial separator and LiTFSI based electrolyte. As the lithium polysulfide migration has been pointed out as major reason for SEI formation and electrolyte decomposition, further optimization in hindering the migrating polysulfide species would directly increase the cycling stability of this Li/S battery setup. Additionally the 'Pechini' precursor developed for versatile Au nanoparticle formation on various substrates allows a transfer of SiNW growth to other 3D substrates with optimized weight and porosity to further reduce the amount of electrolyte and an increased energy density of fully integrated Li/S battery cells for electro mobile applications.

In summary, this publication shows a new set of material and cell concepts for the Li/S battery system with high energy densities and long cycling stability with absence of elemental Li foil. Cathodes with high sulfur content made of carbon composites with a defined porosity ( $>65 \mathrm{wt}$. $\%$ in carbon) and high specific capacity above $1200 \mathrm{mAh} / \mathrm{g}_{\text {sulfur }}(1000 \mathrm{mAh} / \mathrm{g}$ after 100 cycles) were prepared. The versatile gold precursor developed for a homogenous Au layer deposition allowed an adjustable Si loading to achieve balanced SiNW/C composite electrodes. The CVD SiNW growth occurred directly on the surface of the carbon fiber with a high area capacity of up to $9 \mathrm{mAh} / \mathrm{cm}^{2}\left(4 \mathrm{mAh} / \mathrm{cm}^{2}\right.$ after 50 cycles $)$. The result was a SiNW Li/S full-cell with an areal capacity of $2.4 \mathrm{mAh} / \mathrm{cm}^{2}$, a cyclability surpassing 450 cycles and capacity retention of $80 \%$ after 150 cycles (capacity loss lower than $0.4 \%$ per cycle). A low degradation due to a low self-reduction of polysulfides during charging/discharging is revealed with detailed in-operando synchrotron measurements, while other fading mechanisms could be excluded in the SiNW Li/S full-cell setup. The integration of SiNWs on carbon meshes is a promising approach for achieving high capacity batteries in conjunction with sulfur cathodes to avoid dendrite formation and gain improved cycling stability.

\section{Methods}

SiNW/carbon composite electrode preparation. Commercially available carbon fiber networks were used as a three-dimensional conducting substrate for SiNW deposition (Freudenberg FCCT SE \& Co. KG, area weight $6.5 \mathrm{mg} / \mathrm{cm}^{2}$, size $\left.3 \times 4 \mathrm{~cm}^{2}\right)^{22}$. A slightly modified noble metal 'Pechini' precursor based on citric acid, ethylene glycol and $\mathrm{HAuCl}_{4}$ solution was applied for conformal deposition of a thin layer of Au on the carbon mesh fibers (see Suppl. Info. for detailed description).

In order to remove all organic residues that could potentially contaminate the catalyst for successful nanowire growth, the substrates with Au nanoparticles where exposed to pure $\mathrm{H}_{2}$ prior to SiNW growth for $5 \mathrm{~min}$ at $420^{\circ} \mathrm{C}$ in a chemical vapor deposition (CVD) vacuum furnace (ATV GmbH, PEO603). The SiNWs were subsequently grown at a temperature of $420^{\circ} \mathrm{C}$ with a $\mathrm{H}_{2}: \mathrm{SiH}_{4}$ gas flow mixture of $10: 1$ and at a pressure of $150 \mathrm{mbar}$ for $40 \mathrm{~min}$.

Positive electrode preparation. For the positive electrode, carbon as a porous conducting sulfur host helps to overcome the electrically insulating nature of the active material species $\left(\mathrm{S}_{8}, \mathrm{Li}_{2} \mathrm{~S}_{2} / \mathrm{Li}_{2} \mathrm{~S}\right)^{50}$. The sulfur loading was increased up to $65 \mathrm{wt}$.\% in a S/C composite stack with long cycling duration ${ }^{51}$. The cathodes were prepared by a slightly adapted procedure from Bauer et al. ${ }^{52}$ mixing sulfur (Sigma Aldrich, 99.5\%), conductive carbon black (Orion carbon) and mild grinding in a mortar mill (Fritsch Pulverisette 2) for $10 \mathrm{~min}$. Subsequently, a water-based binder solution of carboxymethyl cellulose (CMC, MTI Corporation) and styrene-butadiene rubber (SBR, Targray) (ratio CMC:SBR $=1: 1, \mathrm{~m} / \mathrm{m}$ ) was added and the slurry was again treated for $15 \mathrm{~min}$ in the mill. The weight ratio of sulfur:carbon:CMC:SBR in the resulting slurry was 65:30:2.5:2.5. The slurry was casted onto 


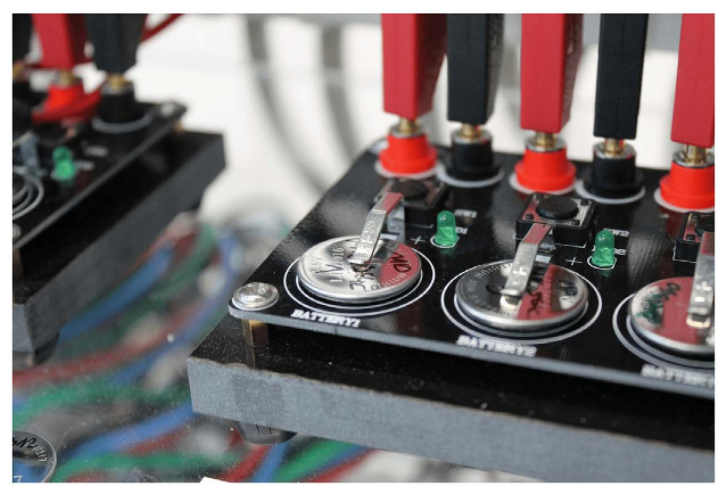

Figure 12. Battery cell test setup for electrochemical characterization of coin cells.

aluminum foil (MTI Corporation, $>99.9 \%$ ) via the doctor-blade coating technique. Drying of the as-prepared positive electrode sheets consisted of two steps, heating with $2{ }^{\circ} \mathrm{C} / \mathrm{min}$ to $80^{\circ} \mathrm{C}$ and thermal treatment at $80^{\circ} \mathrm{C}$ for $1.5 \mathrm{~h}$.

Cell Assembly and electrochemical testing. Both half and full-cells with SiNW/C composite electrodes (diameter $12 \mathrm{~mm}$ ) have been prepared and characterized. Half-cell setups are assembled as coin cells (MTI Corp., CR2016) in an argon-filled glove box (MBraun, $<0.1$ ppm $\mathrm{O}_{2}$ and $\mathrm{H}_{2} \mathrm{O}$ ). Metallic lithium (Pi-Kem, 99.0\%, diameter $15.6 \mathrm{~mm}$, thickness $250 \mu \mathrm{m}$ ) was used as negative electrode. Due to the respective potential vs. $\mathrm{Li} / \mathrm{Li}^{+}$, the SiNW/C composite acts as positive electrode. The electrodes were separated by one layer of a polypropylene sheet (Celgard 2500, diameter $19 \mathrm{~mm}$ ). The electrolyte consisted of $1 \mathrm{M}$ lithium bis-(trifluoromethyl-sulfonyl)-imide (LiTFSI, Aldrich, 99.95\%), 0.25 $\mathrm{M}$ lithium nitrate $\left(\mathrm{LiNO}_{3}\right.$, Alfa Aesar, 99.98\%, anhydrous) in 1,2-dimethoxy ethane (DME, Sigma Aldrich, 99.5\%, anhydrous) and 1,3-dioxolane (DOL, Sigma Aldrich, 99.8\%, anhydrous). The ratio of the solvents was 1:1 (v/v). The cells were cycled at room temperature with a BaSyTec Cell Test System (CTS). Figure 12 shows the battery in the test system. The lower and upper cut-off voltage was set to 0.01 and $1.0 \mathrm{~V}$ vs. $\mathrm{Li} / \mathrm{Li}^{+}$, respectively. Due to the unknown maximum capacity, a constant area current of $0.5 \mathrm{~mA} / \mathrm{cm}^{2}$ was used to evaluate the available capacity. $40 \mu \mathrm{l}$ of the electrolyte were added to all coin cells. All chemicals except for LiTFSI, DME, and DOL were used as received. To remove residual water, LiTFSI was dried at $120^{\circ} \mathrm{C}$ under vacuum for $24 \mathrm{~h}$ before use. DME and DOL were dried and stored over a $3 \AA$ molecular sieve. All samples exhibited a Coulomb efficiency above $98 \%$ until cell failure.

For the Li/S full-cell setup, the SiNW/C composite electrode was prelithiated within two full cycles and subsequently discharged in coin cells according to the testing conditions described above. The prelithiated SiNW/C composite electrode was disassembled and rinsed with a solvent mixture of DME/DOL 1:1 (v/v) and reassembled as negative electrode vs. the sulfur/carbon composite positive electrode ( $65 \mathrm{wt} . \%$ sulfur content, diameter $12 \mathrm{~mm})$ using the same electrolyte $\left(12 \mu \mathrm{l} / \mathrm{mg}_{\text {sulfur }}\right)$ and separator taken for the previously described half-cells. Due to the potential of the lithiated silicon anode, the cut-off-voltages were changed to 1.3 and 2.6 V. Galvanostatic cycling with potential limitation was carried out at a current of $1 / 5 \mathrm{C}(1 \mathrm{C}$ means full charging in $1 \mathrm{~h})$ at room temperature.

Physical characterization methods. The SiNW/C composite electrode was investigated by electron microscopy, X-ray diffraction and energy dispersive X-ray spectroscopy before and after integration into a battery stack in order to identify the mechanisms causing the capacity fade during cycling. Scanning electron micrographs (SEM) where taken on a ZEISS GEMINI LEO 1560 with Bruker in-lens detector, $5 \mathrm{kV}$ acceleration voltage and a typical operating distance of $1 \mathrm{~cm}$. X-ray powder diffraction (XRD) experiments were performed in transmission geometry with Co $\mathrm{K}_{1}$ radiation on an STOE Stadi P diffractometer with curved Ge(111) crystal monochromator and $6^{\circ}$-position sensitive detector. The Rietveld analysis was performed with the program MAUD ${ }^{53}$ assuming an isotropic size distribution. The Popa line broadening model was applied to determine the size and strain of the crystalline phases.

TEM investigations were performed with a FEI Tecnai F30 equipped with field emission gun and operated at $300 \mathrm{kV}$. Energy dispersive X-ray (EDXS) spectroscopy was operated in scanning mode (STEM). The selective area electron diffraction (SAED) pattern was analyzed with the program ELDISCA. For the cycled sample, a diluted $\mathrm{HNO}_{3}$ solution has been used to remove residuals from the electrolyte as well as the solid electrolyte interface (SEI). Prior to analysis, the samples were gently grinded and dispersed in acetone. The dispersion was dropped onto a copper mesh coated with lacey carbon film for TEM analysis.

In-operando synchrotron XRD. In-operando XRD measurements have been done at the beamline P02.1 of the 3rd generation Synchrotron Radiation Source PETRA III at DESY in Hamburg. In an 8-fold coin cell holder $^{54,55}$, a modified coin cell (CR2025) with a self-made Kapton window on both electrode sides was used for the in-operando XRD measurements. Figure 13 shows the complete setup including the 8 -fold coin cell holder. To avoid detection of inactive parts of the cell a hole was implemented in the stainless steel spacer. Only the alumina current collector on the cathode is detected as inactive part of the cell. The lithiated SiNWs were fabricated by discharging to $0.01 \mathrm{vs} \mathrm{Li} / \mathrm{Li}^{+}$at $0.5 \mathrm{~mA}$ and assembled vs. the $\mathrm{S} / \mathrm{C}$ cathode. The beam energy was fixed to $60 \mathrm{keV}$, 


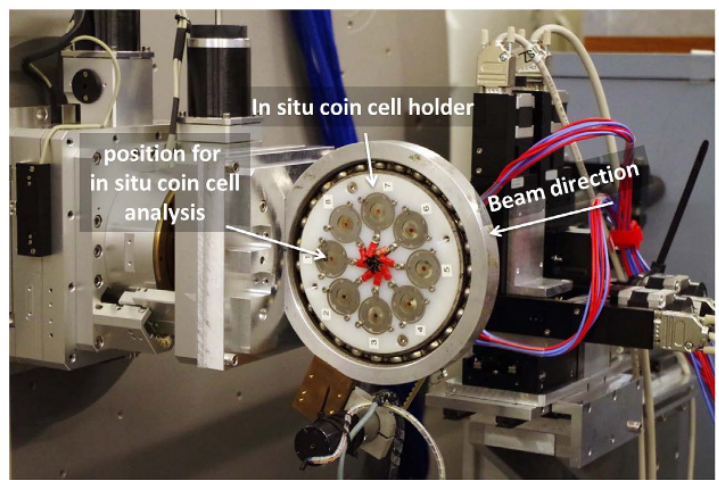

Figure 13. Setup for in-operando synchrotron XRD measurements at DESY Hamburg.

which ensures negligible polarization effects and very low mass absorption coefficients for most elements. A 16-inch two-dimensional flat panel detector of the XRD 1621 N ES Series (PerkinElmer, $2048 \times 2048$ pixels, pixel size $200 \mu \mathrm{m}$ ) was used for recording the diffraction patterns. The exposure time was set to $30 \mathrm{~s}$ and a previously made $30 \mathrm{~s}$ dark image. Detector calibration and two-dimensional image integration were realized by a LaB ${ }_{6} \mathrm{NIST}$ standard and the software Fit $2 D^{56}$. Further details about the setup, the beam optics, the monochromators and experimental possibilities at the high-energy beamlines at $\mathrm{P} 02$ are reported elsewhere ${ }^{57}$.

\section{References}

1. Chen, Lin \& Shaw, Leon L. Recent advances in lithium-sulfur batteries. J. Power Sources 267, 770-783 (2014).

2. Obrovac, M. N. \& Christensen, L. Structural Changes in Silicon Anodes during Lithium Insertion/Extraction. Electrochem. SolidState Lett. 7, A93-A96 (2004).

3. Liang, B., Liu, Y. \& Xu, Y. Silicon-based materials as high capacity anodes for next generation lithium ion batteries. J. Power Sources 267, 469-490 (2014).

4. Jaumann, T. et al. Tailoring Hollow Silicon-Carbon Nanocomposites As High-Performance Anodes in Secondary Lithium-Based Batteries through Economical Chemistry. Chem. Mater. 27, 37-43 (2015).

5. Ma, D., Cao, Z. \& Hu, A. Si-Based Anode Materials for Li-Ion Batteries: A Mini Review. Nano-Micro Lett. 6, 347-358 (2014).

6. Lee, S.-K. et al. Highly Cyclable Lithium-Sulfur Batteries with a Dual-Type Sulfur Cathode and a Lithiated Si/SiOx Nanosphere Anode. Nano Lett. 15, 2863-2868 (2015).

7. Yang, Y. et al. New Nanostructured Li2S/Silicon Rechargeable Battery with High Specific Energy. Nano Lett. 10, 1486-1491 (2010).

8. Liu, N., Hu, L., McDowell, M. T., Jackson, A. \& Cui, Y. Prelithiated Silicon Nanowires as an Anode for Lithium Ion Batteries. ACS Nano 5, 6487-6493 (2011).

9. Chakrapani, V., Rusli, F., Filler, M. A. \& Kohl, P. A. Silicon nanowire anode: Improved battery life with capacity-limited cycling. J. Power Sources 205, 433-438 (2012).

10. Cho, J.-H. \& Picraux, S. T. Silicon Nanowire Degradation and Stabilization during Lithium Cycling by SEI Layer Formation. Nano Lett. 14, 3088-3095 (2014).

11. Ogata, K. et al. Revealing lithium-silicide phase transformations in nano-structured silicon-based lithium ion batteries via in situ NMR spectroscopy. Nat. Commun. 5, 3217, doi: 10.1038/ncomms4217 (2014).

12. Krause, A. et al. Stability and Performance of Heterogeneous Anode Assemblies of Silicon Nanowires on Carbon Meshes for Lithium-Sulfur Battery Applications. In Symposium LL - Semiconductor Nanowires-Growth, Physics, Devices and Applications 1751, doi: 10.1557/opl.2015.196 (2015).

13. Qu, Y., Zhou, H. \& Duan, X. Porous silicon nanowires. Nanoscale 3, 4060-4068 (2011).

14. Hagen, M. et al. Studies on preventing Li dendrite formation in Li-S batteries by using pre-lithiated Si microwire anodes. J. Power Sources 248, 1058-1066 (2014).

15. Lu, C. et al. Core-shell CNT-Ni-Si nanowires as a high performance anode material for lithium ion batteries. Carbon 63, 54-60 (2013).

16. Wu, H. et al. Stable cycling of double-walled silicon nanotube battery anodes through solid-electrolyte interphase control. Nat. Nanotechnol. 7, 310-315 (2012).

17. Chou, S.-L. et al. Silicon/Single-Walled Carbon Nanotube Composite Paper as a Flexible Anode Material for Lithium Ion Batteries. J. Phys. Chem. C 114, 15862-15867 (2010).

18. Forney, M. W. et al. High performance silicon free-standing anodes fabricated by low-pressure and plasma-enhanced chemical vapor deposition onto carbon nanotube electrodes. J. Power Sources 228, 270-280 (2013).

19. Forney, M. W., Ganter, M. J., Staub, J. W., Ridgley, R. D. \& Landi, B. J. Prelithiation of Silicon-Carbon Nanotube Anodes for Lithium Ion Batteries by Stabilized Lithium Metal Powder (SLMP). Nano Lett. 13, 4158-4163 (2013)

20. Kim, H. J. et al. Controlled Prelithiation of Silicon Monoxide for High Performance Lithium-Ion Rechargeable Full Cells. Nano Lett. 16, 282-288 (2016).

21. Peled, E. et al. Tissue-like Silicon Nanowires-Based Three-Dimensional Anodes for High-Capacity Lithium Ion Batteries. Nano Lett. 15, 3907-3916 (2015)

22. Brückner, J. et al. Carbon-Based Anodes for Lithium Sulfur Full Cells with High Cycle Stability. Adv. Funct. Mater. 24, 1284-1289 (2014).

23. Jha, H., Buchberger, I., Cui, X., Meini, S. \& Gasteiger, H. A. Li-S Batteries with Li2S Cathodes and Si/C Anodes. J. Electrochem. Soc. 162, A1829-A1835 (2015).

24. Krause, A., Grube, M., Mikolajick, T. \& Weber, W. M. Comparison of Silicon Nanowire Growth on SiO2 and on Carbon Substrates. ECS Trans. 70, 69-78 (2015).

25. Hagen, M. et al. Lithium-Sulfur Cells: The Gap between the State-of-the-Art and the Requirements for High Energy Battery Cells. Adv. Energy Mater. 5, doi: 10.1002/aenm.201401986 (2015).

26. Chan, C. K. et al. High-performance lithium battery anodes using silicon nanowires. Nat. Nanotechnol. 3, 31-35 (2008).

27. Schmidt, V., Wittemann, J. V., Senz, S. \& Gösele, U. Silicon Nanowires: A Review on Aspects of their Growth and their Electrical Properties. Adv. Mater. 21, 2681-2702 (2009). 
28. Aurbach, D. et al. Nanoparticles of SnO Produced by Sonochemistry as Anode Materials for Rechargeable Lithium Batteries. Chem. Mater. 14, 4155-4163 (2002).

29. Seong, I. W., Hong, C. H., Kim, B. K. \& Yoon, W. Y. The effects of current density and amount of discharge on dendrite formation in the lithium powder anode electrode. J. Power Sources 178, 769-773 (2008).

30. Szczech, J. R. \& Jin, S. Nanostructured silicon for high capacity lithium battery anodes. Energy Env. Sci 4, 56-72 (2011).

31. Kim, H. \& Cho, J. Superior Lithium Electroactive Mesoporous Si@Carbon Core-Shell Nanowires for Lithium Battery Anode Material. Nano Lett. 8, 3688-3691 (2008).

32. Etacheri, V. et al. Exceptional Electrochemical Performance of Si-Nanowires in 1,3-Dioxolane Solutions: A Surface Chemical Investigation. Langmuir 28, 6175-6184 (2012).

33. Lacey, M. J., Edström, K. \& Brandell, D. Visualising the problems with balancing lithium-sulfur batteries by 'mapping' internal resistance. Chem Commun 51, 16502-16505 (2015).

34. Cui, L.-F., Hu, L., Choi, J. W. \& Cui, Y. Light-Weight Free-Standing Carbon Nanotube-Silicon Films for Anodes of Lithium Ion Batteries. ACS Nano 4, 3671-3678 (2010).

35. Obrovac, M. N. \& Krause, L. J. Reversible Cycling of Crystalline Silicon Powder. J. Electrochem. Soc. 154, A103-A108 (2007).

36. Li, J. \& Dahn, J. R. An in situ X-Ray Diffraction Study of the Reaction of Li with Crystalline Si. J. Electrochem. Soc. 154, A156-A161 (2007).

37. Gu, M. et al. Electronic Origin for the Phase Transition from Amorphous LixSi to Crystalline Li15Si4. ACS Nano 7, 6303-6309 (2013).

38. Misra, S. et al. In situ X-ray Diffraction Studies of (De)lithiation Mechanism in Silicon Nanowire Anodes. ACS Nano 6, 5465-5473 (2012).

39. Rohrer, J. \& Albe, K. Insights into Degradation of Si Anodes from First-Principle Calculations. J. Phys. Chem. C 117, 18796-18803 (2013).

40. Zhang, S. et al. Nickel Nanocone-Array Supported Silicon Anode for High-Performance Lithium-Ion Batteries. Adv. Mater. 22, 5378-5382 (2010)

41. Liu, X. H. et al. Self-Limiting Lithiation in Silicon Nanowires. ACS Nano 7, 1495-1503 (2013).

42. Wang, W. et al. Binder-free three-dimensional silicon/carbon nanowire networks for high performance lithium-ion battery anodes. Nano Energy 2, 943-950 (2013).

43. Zhang, S. S. Liquid electrolyte lithium/sulfur battery: Fundamental chemistry, problems, and solutions. J. Power Sources 231, 153-162 (2013)

44. Song, M.-K., Cairns, E. J. \& Zhang, Y. Lithium/sulfur batteries with high specific energy: old challenges and new opportunities. Nanoscale 5, 2186-2204 (2013).

45. Barchasz, C. et al. Lithium/Sulfur Cell Discharge Mechanism: An Original Approach for Intermediate Species Identification. Anal. Chem. 84, 3973-3980 (2012).

46. Waluś, S. et al. New insight into the working mechanism of lithium-sulfur batteries: in situ and operando X-ray diffraction characterization. Chem. Commun. 49, 7899-7901 (2013).

47. Cañas, N. A., Wolf, S., Wagner, N. \& Friedrich, K. A. In-situ X-ray diffraction studies of lithium-sulfur batteries. J. Power Sources 226, 313-319 (2013).

48. Kulisch, J., Sommer, H., Brezesinski, T. \& Janek, J. Simple cathode design for Li-S batteries: cell performance and mechanistic insights by in operando X-ray diffraction. Phys. Chem. Chem. Phys. 16, 18765-18771 (2014).

49. Waluś, S. et al. Lithium/Sulfur Batteries Upon Cycling: Structural Modifications and Species Quantification by In Situ and Operando X-Ray Diffraction Spectroscopy. Adv. Energy Mater. 5, 1500165 (2015).

50. Strubel, P. et al. ZnO Hard Templating for Synthesis of Hierarchical Porous Carbons with Tailored Porosity and High Performance in Lithium-Sulfur Battery. Adv. Funct. Mater. 25, 287-297 (2015).

51. Thieme, S. et al. High capacity micro-mesoporous carbon-sulfur nanocomposite cathodes with enhanced cycling stability prepared by a solvent-free procedure. J. Mater. Chem. A 1, 9225-9234 (2013).

52. Bauer, I., Thieme, S., Brückner, J., Althues, H. \& Kaskel, S. Reduced polysulfide shuttle in lithium-sulfur batteries using Nafionbased separators. J. Power Sources 251, 417-422 (2014).

53. Lutterotti, L., Chateigner, D., Ferrari, S. \& Ricote, J. Texture, residual stress and structural analysis of thin films using a combined $\mathrm{X}$-ray analysis. Thin Solid Films 450, 34-41 (2004).

54. Herklotz, M., Weiss, J., Giebeler, L. \& Knapp, M. Inventors; Karlsruher Institut für Technologie, Leibniz-Institut für Festkörper- und Werkstoffforschung Dresden e.V. assignee. Batterieträger. Germany patent DE 102,014,211,901. Mar 19 (2015).

55. Herklotz, M. et al. A novel high-throughput setup for in situ powder diffraction on coin cell batteries. J. Appl. Crystallogr. 49, 340-345 (2016)

56. Hammersley, A. P., Svensson, S. O., Hanfland, M., Fitch, A. N. \& Hausermann, D. Two-dimensional detector software: From real detector to idealised image or two-theta scan. High Press. Res. 14, 235-248 (1996).

57. Liermann, H.-P. et al. The Extreme Conditions Beamline at PETRA III, DESY: Possibilities to conduct time resolved monochromatic diffraction experiments in dynamic and laser heated DAC. J. Phys. Conf. Ser. 215, 12029 (2010).

\section{Acknowledgements}

We gratefully thank the Federal Ministry of Education and Research (BMBF) for the financial support within the network project of the WING center BamoSa (FKZ: 03X4637). In-operando XRD was carried out at the synchrotron light source PETRA III at DESY/Hamburg. We would like to thank Alexander Schökel for assistance in using beamline P02.1, Liuda Mereacre (KIT) for modified cell preparation and Geethu Balachandran (KIT) for help with the sample preparation. We acknowledge support by the German Research Foundation and the Open Access Publication Funds of the TU Dresden.

\section{Author Contributions}

Dr. A.K.: SiNW growth and optimization, physical characterization (SEM, XRD), AuNP preparation, characterization planning, discussion and writing. Dr. S.D.: Sulfur/Carbon cathode preparation, Precursor development, discussion and writing. MSc M.P.: Cell assembly, electrochemical characterization of half- and full-cells and writing. Dr. F.M.W.: Precursor development, Substrate preparation with Au NPs and writing. MSc T.J.: XRD, TEM, in-operando synchrotron XRD, discussion and writing. Dr. E.A.: in-operando synchrotron XRD, cell preparation, writing. Dr. L.G.: in-operando synchrotron XRD, discussion and writing. Dr. H.A.: Scientific planning of electrochemical experiments of Li/S cells. Dr. S.S.: Scientific support and discussion. Dr. J.G.: Scientific support and discussion for Au precursor development. MSc A.J.: SiNW anode preparation, electrochemical analysis, writing. Dr. M.G.: SiNW growth, scientific discussion. Dr. J.B.: Cell assembly, electrochemical characterization of half-cells. MSc J.M.: Precursor development and optimization. Prof. J.E.: 
Scientific planning and discussion of physical characterization of Li battery cells. Prof. S.K.: Scientific planning of electrochemical analysis and sulfur cathode preparation. Prof. T.M.: Scientific planning and discussion of SiNW implementation. Dr. W.M.W.: Scientific planning and discussion of SiNW growth optimization, discussion and writing.

\section{Additional Information}

Supplementary information accompanies this paper at http://www.nature.com/srep

Competing financial interests: The authors declare no competing financial interests.

How to cite this article: Krause, A. et al. High Area Capacity Lithium-Sulfur Full-cell Battery with Prelitiathed Silicon Nanowire-Carbon Anodes for Long Cycling Stability. Sci. Rep. 6, 27982; doi: 10.1038/srep27982 (2016).

(c) (i) This work is licensed under a Creative Commons Attribution 4.0 International License. The images Co other third party material in this article are included in the article's Creative Commons license, unless indicated otherwise in the credit line; if the material is not included under the Creative Commons license, users will need to obtain permission from the license holder to reproduce the material. To view a copy of this license, visit http://creativecommons.org/licenses/by/4.0/ 